\title{
Breathless cancer cells get fat on glutamine
}

\author{
Dimitrios Anastasiou ${ }^{1}$, Lewis C Cantley ${ }^{1}$ \\ ${ }^{1}$ Beth Israel Deaconess Medical Center, Department of Medicine-Division of Signal Transduction; Department of Systems Biology, \\ Harvard Medical School, Boston, MA 02115, USA \\ Cell Research (2012) 22:443-446. doi:10.1038/cr.2012.5; published online 3 January 2012
}

\begin{abstract}
Many cancer cells depend on glutamine as a fuel for proliferation, yet the mechanisms by which glutamine supports cancer metabolism are not fully understood. Two recent studies highlight an important role for glutamine in the synthesis of lipids and provide novel insights into how glutamine metabolism could be targeted for cancer therapy.
\end{abstract}

As a consequence of rapid proliferation cancer cells require a constant supply of building blocks to generate daughter cells. It is increasingly appreciated that most oncogenic pathways lead to changes in the cancer proteome that facilitate nutrient uptake and reprogram metabolic processes to promote the utilization of nutrients for anabolism [1]. A boost in lipid synthesis is critical for supporting proliferation because, in addition to serving signaling functions, lipids are essential structural components of cellular membranes. Cancer cells prefer to generate lipids de novo rather than use them directly from their environment [2]. It has been proposed that glucose can provide carbon atoms for lipid synthesis in cancer cells. Two studies by Metallo et al.[3] and Mullen et al.[4] shed fresh light into how another key nutrient for cancer, glutamine, also contributes to lipogenesis.

\footnotetext{
Correspondence: Lewis C Cantley

Tel: +1 617735 2601; Fax: +1 6177352646

E-mail: lewis_cantley@hms.harvard.edu
}

Glucose is predominantly metabolized through a series of enzymatic reactions collectively known as glycolysis to generate pyruvate. Pyruvate is imported into mitochondria where it is converted to acetyl-coenzyme A (acetyl$\mathrm{CoA}$ ) by the pyruvate dehydrogenase (PDH) enzyme complex (Figure 1A). Acetyl-CoA then condenses with oxaloacetate to enter the tricarboxylic acid cycle (TCA cycle, also known as the Krebs cycle or citric acid cycle), which provides reducing power to fuel energy generation in the form of ATP via the oxidative phosphorylation (OXPHOS) chain. Alternatively, pyruvate is converted to lactate by cytoplasmic lactate dehydrogenase (LDH). In cancer cells the relative amount of pyruvate oxidized to lactate versus that metabolized via the TCA cycle is higher compared to normal cells. However, in many cancer cells, a fraction of glucose-derived acetyl-CoA is also incorporated into lipids. This is because mitochondrial citrate exits into the cytoplasm, where the enzyme ATP citrate lyase (ACL) converts it to acetylCoA and oxaloacetate [5]. Cytoplasmic acetyl-CoA can then be utilized for lipid synthesis.

Glutamine has long been recognized as an essential nutrient for the proliferation of most cancer cells [6] but also untransformed cells in culture. In addition to being utilized for protein synthesis, glutamine also serves as a precursor for other amino acids [7]. Furthermore, glutamine provides nitrogen for the synthesis of nucleotide bases, and deoxyribonucleotide supplementation can rescue a cell cycle arrest caused by glutamine deprivation in transformed cells [8]. However, in some cancer cell lines, glutamine can be consumed at rates that exceed the need for nitrogen provision [9]. One route of glutamine catabolism involves its conversion to glutamate by glutaminase (GLS) in mitochondria. Glutamate is, in turn, deaminated to generate $\alpha$-ketoglutarate $(\alpha-K G), \alpha-K G$ can then enter the TCA cycle and keep it going when glucosederived citrate is re-routed to the cytoplasm. This function of glutamine is referred to as "anaplerosis" (which means "to top up").

Under hypoxic conditions, which are frequently encountered in the tumor environment, pyruvate enters mitochondria at reduced rates. This is, in part, because hypoxia induces the stabilization of the transcription factor HIF-1a (hypoxia-inducible factor-1a). One of the transcriptional targets of HIF-1a is pyruvate dehydrogenase kinase 1 (PDK1), which limits conversion of pyruvate into acetyl-CoA in mitochondria by phosphorylating the E1 subunit of $\mathrm{PDH}$ and thus inactivating the enzyme. Limiting pyruvate entry into mitochondria will also reduce glucose-derived carbons available for acetyl-CoA and ultimately lipid synthesis. This raises the question: what is the source of carbon for lipid synthesis under hypoxia? 
Interestingly, the dependence on glutamine for proliferation persists even under hypoxic conditions, against the prediction that limited activity of the respiratory chain should alleviate the need for anaplerosis. Metallo et al. [3] observed that cells cultured under hypoxia took up glutamine at higher rates than under normoxia and use it for lipid synthesis. To gain insights into how glutamine contributes to lipogenesis, they followed the fate of glutamine in various cell lines. To this end, they utilized glutamine that harbored ${ }^{13} \mathrm{C}$ instead of the naturally most abundant carbon isotope ${ }^{12} \mathrm{C}$. As various intracellular metabolites incorporated ${ }^{13} \mathrm{C}$ from glutamine, their mass increased proportionally to the number of glutamine-derived carbons. Such mass increases can be accurately monitored by mass spectrometry. Importantly, by replacing select carbons in glutamine with ${ }^{13} \mathrm{C}$, information about the route that each ${ }^{13} \mathrm{C}$ atom takes to end up in a given metabolite can be deduced. Using this approach, Metallo et al. found that rather than generating citrate going through the forward, oxidative, direction of the TCA cycle, glutamine utilizes another route that involves the reductive carboxylation of glutaminederived $\alpha-K G$ (Figure 1B). As much as $25 \%$ of acetyl-CoA utilized for lipid synthesis was generated by this route of glutamine catabolism in various cell lines under normoxic conditions, the rest of the carbons being contributed by glucose.

When they probed the source of lipogenic acetyl-CoA under hypoxia, Metallo et al. observed that less glucose is used for lipid synthesis, consistent with the notion that hypoxia limits pyruvate entry into mitochondria. Under these conditions, however, glutamine took over the role of the major carbon source for lipogenesis via the reductive carboxylation route. Treatment of cells with dichloroacetate (DCA), which inhibits PDK1 and therefore alleviates inhibition of PDH (Figure 1B), partly restored glucose-dependent lipogenesis and diminished reductive glutamine metabolism under hypoxia. Attesting to the role of HIFs in dictating reductive glutamine metabolism, Metallo et al. investigated the fate of glutamine in renal cell carcinoma (RCC) cell lines that lack the von Hippel Lindau (VHL) tumor suppressor. VHL targets HIF for degradation under normoxia. In these cell lines, HIF is constitutively expressed

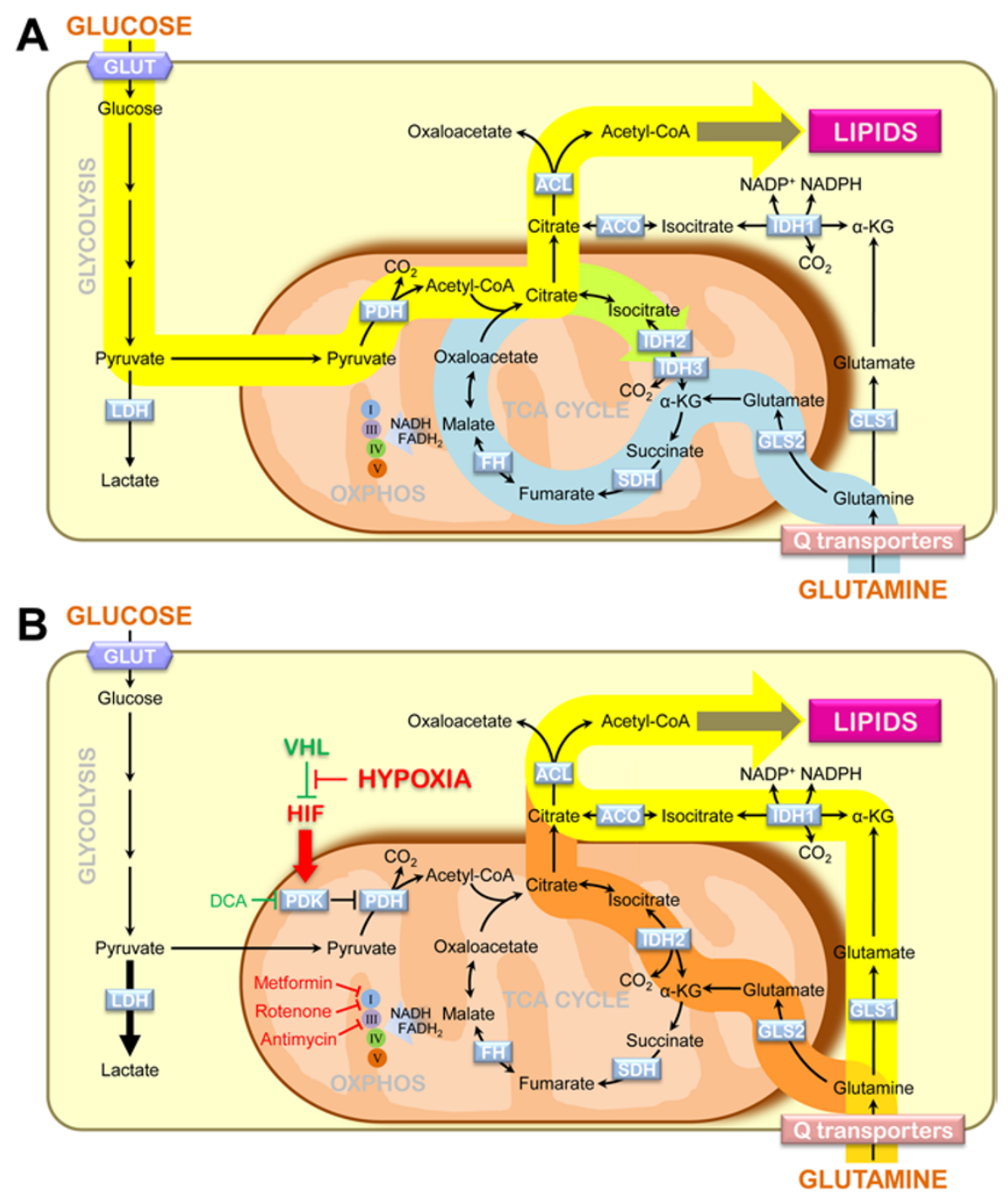

Figure $1 \mathrm{~A}$ metabolic switch in carbon source for lipid synthesis. (A) Under conditions when mitochondrial respiration is fully functional, glucose is the major source of carbons for acetyl-CoA to synthesize lipids. The yellow block arrow indicates the route of carbons from glucose to lipids. The blue block arrow indicates oxidative glutamine metabolism (anaplerosis). The green block arrow shows the metabolic pathway of citrate generated from glucose-derived acetyl-CoA and glutamine-derived oxaloacetate. (B) Under conditions where HIF is stabilized (such as hypoxia or upon VHL deletion) or when mitochondrial respiration is limited, glutamine predominantly provides carbons for lipogenic acetyl-CoA either via cytoplasmic IDH1 (yellow block arrow) or mitochondrial IDH2 (orange block arrow). This pathway is referred to as reductive glutamine metabolism. The exact route of glutamine to acetyl-CoA most likely depends on the extend of mitochondrial respiration. In red and green are processes or compounds that promote or prevent reductive glutamine metabolism, respectively. Key enzymes are represented in blue boxes and their name abbreviations are described in the text. 
even under normoxic conditions. In contrast to cells with intact VHL where most of lipogenic acetyl-CoA originated from glucose, in VHL-mutated RCC cells up to $70 \%$ of lipid carbons were derived from glutamine. When VHL expression was restored in these cells, lipid synthesis switched back to glucose as the main carbon source.

Despite early hypotheses that defective mitochondria underlie the characteristic glucose metabolism exhibited by cancer cells, in most cancers mitochondrial function is intact [1]. However, some cancers harbor mutations in genes encoding mitochondrial proteins that lead to aberrant mitochondrial metabolism. In such cancers, glucose-dependent acetyl-CoA generation and lipid synthesis would also be compromised. To understand how cancer cells with mitochondrial defects generate biosynthetic precursors, Mullen et al. [4] generated a pair of isogenic cell lines where mitochondria had been previously depleted and subsequently re-populated using wild-type mitochondrial DNA or mitochondrial DNA containing a mutation in cytochrome $\mathrm{c}$ that abrogated mitochondrial respiration. Indeed, glucose-dependent lipogenesis was severely impaired in cells with defective mitochondria. Interestingly, both cell lines required glutamine for anchorage-independent growth, suggesting that glutamine supports proliferation by a respirationindependent pathway. Mullen et al. also used ${ }^{13} \mathrm{C}$-labeled glutamine to trace its metabolic fate with mass spectrometry and found that, similar to cells under hypoxia, cells with defective mitochondria generate the majority of lipogenic acetyl-CoA via reductive carboxylation of $\alpha-K G$. This is also the case in RCC cell lines with mutations in the TCA cycle enzyme fumarate hydratase (FH), suggesting that this pathway is also relevant for tumors that naturally harbor mitochondrial defects. Mullen et al. showed that pharmacologic impairment of mitochondrial function by respiratory chain inhibitors (metformin, rotenone or antimycin) (Figure 1B) also induced reductive glutamine metabolism in cells with functional mitochondria, evidence in support of a more general role for reductive glutamine metabolism in cancer cells.

Reductive glutamine metabolism involves the carboxylation of $\alpha-\mathrm{KG}$ by $\mathrm{CO}_{2}$, a reaction that can be performed by some isocitrate dehydrogenase (IDH) isoforms by operating in the reverse direction compared to the classical TCA cycle reaction that they catalyze. In mammalian cells, there are three known IDH isoforms: IDH1 and IDH2 which are both dependent on $\mathrm{NADP}^{+}$and localize to the cytosol and mitochondria, respectively; and IDH3 which depends on $\mathrm{NAD}^{+}$and also localizes to mitochondria. To shed light into the pathway that mediates reductive glutamine metabolism, both Metallo et al. and Mullen et al. used RNA interference to reduce the expression of all three IDHs individually and both groups showed that reductive glutamine metabolism depends on the presence of IDH1. Mullen et al. also observed a dependence of the pathway on IDH2. Given that they studied the role of IDHs in respiration-defective cells, it is likely that, in this setting, the TCA cycle runs significantly slower and leads to accumulation of $\alpha-K G$ that pushes IDH2 to run in the reverse direction by mass action (Figure 1B). While this interpretation could also be applicable to IDH3, this isoform is thought to operate exclusively in the forward, oxidative direction, hence its depletion does not affect reductive glutamine metabolism. Interestingly, an independent study by Wise et al. [10] also pointed to IDH2 as the enzyme that mediates reductive glutamine metabolism under hypoxia. While Wise et al. did not compare the effects of knocking down all three IDH isoforms to exclude a role for IDH1, it is possible that, as they used an even lower oxygen concentration for their experiments than Metallo et al., respiration was limited to levels comparable with those found in the cells used by Mullen et al., hence the similarities in their findings with the latter study. In combination, however, these data suggest that reductive glutamine metabolism can take place in both the cytoplasm and mitochondria, possibly depending on the genetic background or local microenvironment conditions, such as the oxygen concentrations experienced by individual tumors or within different regions of the same tumor. Regardless, consistent with the idea that IDH1 and 2 perform an essential function in providing lipid precursors, knockdown of either isoform hindered cell proliferation $[3,4,10]$.

The studies by Mullen et al. and Metallo et al., as well as that of Wise et $a l$., present compelling evidence that under conditions where glucose carbon influx into mitochondria is limiting, a significant fraction of carbons for lipid synthesis is provided by glutamine through a previously underappreciated pathway. As Metallo et al. propose, by taking over the burden of providing carbons for lipid synthesis, reductive glutamine metabolism frees up glucose carbons to enter into other anabolic pathways. Interestingly, this metabolic pathway is not exclusive to cancer cells as, under hypoxia, non-transformed lung fibroblasts or activated primary $\mathrm{T}$ lymphocytes also exhibited enhanced dependence on reductive glutamine metabolism for lipogenesis [3], suggesting a broader role for proliferative metabolism.

Intriguingly, in addition to its role as a metabolic intermediate in anabolic reactions, acetyl-CoA is also a precursor for protein acetylation. Glucose availability has therefore been proposed to affect histone acetylation and thereby gene expression [11]. It is tempting to speculate that modulation of reductive glutamine metabolism by hypoxia may also have a role in protein acetylation, but whether it is equivalent to that of glucose in that it provides acetyl-CoA 
remains to be investigated.

Idh 1 and Idh 2 mutations have recently been identified in a variety of tumors. These mutations lead to a substitution of an amino acid at the active site of the protein that abrogates activity in the forward, oxidative direction, but allows the enzyme to operate in the reductive direction, albeit modified so that it produces 2-hydroxyglutarate (2-HG) instead of isocitrate $[12,13]$. Mutant IDH1/2 enzymes are therefore attractive targets for developing novel therapeutics for tumors that harbor these mutations. The studies of Mullen et al. and Metallo et al. now suggest that, in addition to other enzymes involved in the pathway such as aconitase (ACO), wild-type IDH1 or IDH2 could also serve as potential candidates for targeting hyperproliferation diseases. Given their role in the TCA cycle, inhibiting specifically the reductive direction of these enzymes would be essential to selectively hinder tumor cell proliferation and this may be challenging. Regardless, by elucidating yet another idiosyncrasy of proliferative metabolism, these studies provide a better grasp of the cancer arsenal and bring us a step closer to understanding how metabolic reprogramming contributes to cancer development.

\section{References}

1 Vander Heiden MG, Cantley LC, Thompson CB. Understanding the Warburg effect: the metabolic requirements of cell proliferation. Science 2009; 324:1029-1033.

2 Ookhtens M, Kannan R, Lyon I, Baker N. Liver and adipose tissue contributions to newly formed fatty acids in an ascites tumor. Am J Physiol 1984; 247:R146-R153.

3 Metallo CM, Gameiro PA, Bell EL, et al. Reductive glutamine metabolism by IDH1 mediates lipogenesis under hypoxia. Nature 2011; 481:380-384.

4 Mullen AR, Wheaton WW, Jin ES, et al. Reductive carboxylation supports growth in tumour cells with defective mitochondria. Nature 2011; 481:385388.

5 Hatzivassiliou G, Zhao F, Bauer DE, et al. ATP citrate lyase inhibition can suppress tumor cell growth. Cancer Cell 2005; 8:311-321.

6 Eagle H. Nutrition needs of mammalian cells in tissue culture. Science 1955; 122:501-514

7 DeBerardinis RJ, Cheng T. Q's next: the diverse functions of glutamine in metabolism, cell biology and cancer. Oncogene 2010; 29:313-324.
8 Gaglio D, Soldati C, Vanoni M, Alberghina L, Chiaradonna F. Glutamine deprivation induces abortive s-phase rescued by deoxyribonucleotides in $\mathrm{k}$ ras transformed fibroblasts. PLoS One 2009; 4:e4715.

9 DeBerardinis RJ, Mancuso A, Daikhin $\mathrm{E}$, et al. Beyond aerobic glycolysis: transformed cells can engage in glutamine metabolism that exceeds the requirement for protein and nucleotide synthesis. Proc Natl Acad Sci USA 2007; 104:19345-19350.

10 Wise DR, Ward PS, Shay JE, et al. Hypoxia promotes isocitrate dehydrogenase-dependent carboxylation of alphaketoglutarate to citrate to support cell growth and viability. Proc Natl Acad Sci USA 2011; 108:19611-19616.

11 Wellen KE, Hatzivassiliou G, Sachdeva UM, Bui TV, Cross JR, Thompson CB. ATP-citrate lyase links cellular metabolism to histone acetylation. Science 2009; 324:1076-1080.

12 Dang L, White DW, Gross S, et al. Cancer-associated IDH1 mutations produce 2-hydroxyglutarate. Nature 2009; 462:739-744.

13 Ward PS, Patel J, Wise DR, et al. The common feature of leukemia-associated IDH1 and IDH2 mutations is a neomorphic enzyme activity converting alpha-ketoglutarate to 2-hydroxyglutarate. Cancer Cell 2010; 17:225-234. 